Debate / Controversy

Consumo Colaborativo y Economía del Bien Común Collaborative Consumption and Economy for the Common Good 
Debate / Controversy

\section{Consumo colaborativo: Las razones de un debate}

\section{Collaborative Consumption: Reasons for a Debate}

\section{Luis Enrique Alonso}

Departamento de Sociología. Universidad Autónoma de Madrid. España/Spain

luis.alonso@uam.es

\section{RESUMEN}

El objetivo de este artículo es presentar el debate actual sobre el concepto de economía colaborativa y su derivado más difundido: el consumo colaborativo. Se realiza en el texto una exposición tanto del auge del tema como de las razones que han explicado su éxito: su relación con la economía de redes, su atractivo para grupos sociales movilizados y su crecimiento como práctica defensiva de consumo durante la crisis financiera contemporánea. Finalmente se hace en este trabajo un análisis sobre los peligros de desnaturalización del concepto y su relación con el espacio de los bienes privados, públicos y comunes, animando a una reflexión no idealizada tanto de las potencialidades como de los límites de las diferentes formas de consumo colaborativo.

Palabras clave: Consumo Colaborativo, Economía Colaborativa, Bienes Comunes, Bienes Públicos, Mercantilización, Prosumidor.

\section{ABSTRACT}

The aim of this article is to present the current debate about the concept of collaborative economy and his more known practice: the collaborative consumption. Also in the text are explained the reasons of the success of this issue: his relation with the economy of networks, his attraction for social mobilized groups and his growth like defensive practices of consumption during the financial contemporary crisis. Finally there are analysed the dangers of denaturalization of the concept and his relation with the space of the private, public and common goods; doing a warning on the need of make a not idealized reflection about the potentials, and also about the limits, of the different forms of collaborative consumption

Keywords: Collaborative Consumption, Collaborative Economics, Common Goods, Public Goods, Commoditization, Prosumer.

\footnotetext{
*Autor para correspondencia / Corresponding author: Luis Enrique Alonso. Departamento de Sociología, Facultad de Ciencias Económicas,
} Universidad Autónoma de Madrid, Av. Tomás y Valiente 5, 28049 Madrid

Sugerencia de cita / Suggested citation: Alonso, L. E. (2017). Consumo colaborativo: las razones de un debate. Revista Española de Sociología, 26 (1), 87-95.

(http://dx.doi.org/10.22325/fes/res.2017.4) 
"No es fácil concebir el concepto que tiene un individuo de su propio interés sin ver a la persona en un contexto social. La relación de una persona con los grupos puede ser muy compleja y lo que una persona ve como su propio interés bien puede estar estrechamente ligado con el de los grupos con que la persona se identifica."

Amartya Sen, (1989: 364)

"El consumo conectado puede llevar a un nuevo régimen de producción y consumo que sea más igualitario, más sostenible y que genere mayor cohesión social. Algunas de las iniciativas descritas (...) parecen conseguir estos objetivos, pero otras pueden reproducir las desigualdades que ya existen, fomentar un tipo de demanda de alto impacto ambiental y terminar subsumidas en el paradigma actual, siendo business as usual si les va bien. El que pase una cosa u otra está determinado por varios factores, como el diseño de la iniciativa, que ésta sea lucrativa o no lucrativa, el tipo de usuarios que atraiga y la naturaleza del servicio que proporciona. (...) Dado lo novedoso del consumo conectado, es difícil prever cómo evolucionará."

Juliet Shor (2014: 9)

\section{INTRODUCCIÓN}

Un nuevo apellido ha venido a asociarse en los últimos tiempos al sustantivo de consumo, es el de lo colaborativo. Así hemos visto emerger prácticas en la vida cotidiana de grupos sociales, no desdeñables en número, que han venido catalogándose como de consumo colaborativo. En muy pocos años ha tomado carta de naturaleza y se ha difundido con inusitada rapidez este concepto de consumo colaborativo ${ }^{1}$ para designar un muy amplio número

1 Es un lugar común atribuirle el término de consumo colaborativo a Roy Algar (2007) en un artículo escrito desde el ámbito del management y la consultoría empresarial, fundamentalmente basado en los clubs de consumidores para conseguir mejores condiciones de compra. El lanzamiento final del concepto lo hace Rachel Botsman, auténtica profeta mediática, e incluso académica del de formas de utilización, uso 0 adquisición coordinada y compartida de un conjunto de bienes y servicios que convencionalmente se habían consumido con carácter individual y excluyente. Este carácter de compartido, esto es, de conectividad consciente y auto-coordinada lo diferencia tanto del consumo privado mercantil — donde el mecanismo de precio radicaliza 0 institucionaliza el individualismo posesivo del comprador-, como del consumo colectivo organizado por las agencias estatales en torno a los bienes públicos con una función de utilidad conjunta y con precios totalmente regulados.

El consumo colaborativo, pues, se asocia en su origen a una larga serie de experiencias de consumo supuestamente no convencional y que tienden a estar encuadradas en un campo semántico común (sostenible, responsable, ético, alternativo, solidario, con causa, orgánico, ecológico, etc.) que, ante todo, refleja que la estricta asignación monetaria mercantil y lucrativa de los recursos es sustituida por mecanismos comunitarios (que no directamente estatales) de intercambio consciente sobre los efectos que tanto para el entorno social, como para el entorno natural tiene el propio hecho de consumir.

\section{UN DESARROLLO ESPECTACULAR}

Con no más de media docena de años en su historia, como término acuñado y como movimiento socialmente reconocible, el consumo colaborativo no deja de ser una de las manifestaciones — si se quiere la más visible y publicitada - de toda una conceptualización mayor: la de la economía colaborativa donde la construcción de experiencias comunes para el acceso y la administración de los recursos escasos es el eje esencial del movimiento.

consumo colaborativo y la economía compartida; merece la pena verse el libro que arme intelectualmente al movimiento: Botsman y Rogers (2010). Otros autores por ejemplo Brändle (2013), encuentran antecedentes mucho más antiguos, en lo que se refiere al desarrollo teórico del consumo colaborativo —Felson y Spaeth (1978) — y lo colocan en una tensión esencial entre la economía del espectáculo y del despilfarro y el eterno retorno de la visión comunitaria, siempre presente en lo social, que clama ahora por la sostenibilidad y lo común. 
En este espacio económico no sólo nos encontramos con la idea de la creación de vías de acceso al disfrute de bienes y servicios que no sea la adquisición privativa de ellos a través de los canales comerciales tradicionales - que sería estrictamente el consumo colaborativo-, sino que nos encontramos formas económicas comunitarias de intercambio de cualificaciones, bancos de tiempo, monedas de uso local, redes cooperativas de reparación, reciclaje y producción artesanal, formas de financiación populares y recíprocas (microcréditos) o modalidades asociativas de gestión de suministros (clubs de compradores, cooperativas de distribución, etc.).

Todo este movimiento tiende a alejarse de la lógica mercantil pura (individual, maximizadora, egoísta) sin entrar en la de los bienes públicos estatales, asignados siguiendo un criterio de decisión política basada en una representación ciudadana indirecta e institucionalmente mediada. La economía colaborativa, en teoría, reclama una implicación directa, personal, recíproca y activa de la ciudadanía en la solución económica de la gestión de sus necesidades, compartiendo y colaborando en la generación de bienes y servicios valorados por públicos próximos, concretos y dispuestos a compartir y por ende a colaborar ${ }^{2}$.

En lo que se refiere específicamente al consumo colaborativo, la idea es conocida en las prácticas informales de la vida cotidiana de multitudes de comunidades históricas, compartir y colaborar, entre vecinos, paisanos compañeros o conciudadanos para optimizar el uso de recursos, ha sido y es habitual en el mundo de la vida de muchos grupos a lo largo de la historia, llegando incluso a mantener 0 crear fórmulas de gestión más comunalistas, autogestionarias, cooperativas 0 comunitarias que han tendido a encontrar su lugar

2 Para una introducción muy completa al tema de la economía en colaboración puede verse el muy completo dossier compilado por la economista Carmen Valor (2024), con artículos de los principales impulsores y estudiosos del tema a nivel nacional e internacional (Juliet Sschor, Julio Gisbert, Lucía del, Moral, Albert Cañigueral, etc.). Referencias necesarias en los últimos debates sobre economía colaborativa, conectada o compartida son las de Belk (2010) y McIntosh (2013). —más 0 menos conflictivo - en la evolución de las sociedades modernas y contemporáneas. Pero también es cierto que el impulso, renovado y magnificado por el neoliberalismo hegemónico actual, de la remercantilización absoluta de lo social, han ido dejando fuera de los usos microeconómicos habituales, incluso de lo considerado como "natural" o "sentido común", cualquier solución de suministro que no sea radicalmente el consumo privado (y privativo), posicional y competitivo, representación material del individualismo posesivo que le sirve como constitución moral.

Del mismo modo que la economía de los bienes públicos — además de ser atacada, recortada y arrinconada por el ascenso de la economía financiera global- había hecho poco por crear vínculos participativos y ciudadanos activos en la toma de decisiones de los consumos colectivos atañen directamente a la población. Este distanciamiento y la burocratización asociada acaban también por crear una crisis de decepción y, por ello, de legitimación de lo estatal —en todos sus niveles - que separaba claramente lo público de lo comunitario y cerraba la posibilidad de encontrar caminos para que la colaboración y la actividad organizativa directa de la ciudadanía se abriese, flexibilizase y acercase a la voluntad población la gestión de lo público ${ }^{3}$.

\section{LAS RAZONES DEL CRECIMIENTO}

Varias han sido las causas que han hecho tomar impulso a esta noción del consumo colaborativo. En primer lugar el incremento de las posibilidades coordinadoras de las redes informáticas, su generalización, su potencia y su eficacia. En segundo lugar,

3 Para el tema de la decepción como proceso de alejamiento de lo público y su papel en la formación de ciclos de interés 0 atonía por el espacio político —asociado además a las formas de comportamiento pautadas del público frente a las instituciones (salida, voz, lealtad) - el clásico indiscutible es evidentemente, Albert Hirschman, sus fascinantes planteamientos se pueden encontrar en una muy útil compilación de sus tesis fundamentales (Hirschman, 2014). Para la crisis de legitimación de las instituciones políticas y económicas sigo siendo imprescindible Habermas (1999). 
las necesidades surgidas en torno a la última gran crisis económica y financiera, así como sus efectos empobrecedores y exclusógenos para muchos grupos y franjas sociales especialmente débiles y vulnerables; empobrecimiento aumentado, si cabe, con las políticas de austeridad y reducción del gasto y los consumos públicos, lo que ha abocado a muchos colectivos a buscar soluciones diferentes para su propia supervivencia. Finalmente, el ascenso de los movimientos sociales y su profundización en discursos alternativos y fundamentalmente comunitarios, como forma de rechazo e indignación de las formas de gestión de la crisis tanto por los gobiernos como por los poderes financieros, lo que ha relanzado el interés y la curiosidad por estilos de vida no convencionales y experiencias no mercantiles (pero tampoco estatales) de satisfacción de las necesidades individuales y colectivas.

En cuanto a las redes informáticas, su impulso ha sido definitivo al fenómeno del consumo colaborativo, por múltiples razones, casi todas ellas fáciles de visualizar. Es evidente que permiten una comunicación instantánea y coordinada entre personas dispuestas a compartir y colaborar; el desarrollo de la actividad p2p (peer to peer) posibilita una red de intercambios de información y transacciones directas de bienes y servicios inusitada y sin parangón con cualquier nivel tecnológico anterior. La sociedad de redes permite una conectividad directa, personal y, con posibilidades, auto-organizativas que multiplica las posibilidades de colaboración o la capacidad de compartir muy por encima del ámbito íntimo local o próximo al que estaba tradicionalmente asociado, Ilevándolo a un nivel prácticamente global. Pero esta red, ha construido poderosos nudos, plataformas coordinadas con orígenes muy diversos — desde la espontaneidad, a la iniciativa innovadora técnica pasando por todo tipo de experiencias comunes desde el altruismo puro y compartido, hasta diversos grados de comercialización lucrativa - que le han dado a estas prácticas colaborativas una cierta garantía pseudoinstitucional del cumplimiento de las condiciones de intercambio. La información cruzada, la confianza mutua y el valor reputacional - concepto fundamental en el consumo colaborativo- por que que genera la transparencia de la red 0 la auto-organización de la plataforma es un activo indispensable para facilitar y entrar en el intercambio mutuo, de tal suerte que no es la coacción normativa, sino el consenso participativo el que crea ese beneficio reputacional y la información positiva que permite mantener la confianza a los que ya interactúan en estos entornos y que anima a entrar a aquellos otros que todavía no habían participado en este tipo de experiencias.

Pero si tanto la organización técnica, como sus posibilidades colaborativas, cooperativas y reputacionales de las tecnologías en red han sido fundamentales para lanzar este tipo de economía compartida; no lo han sido menos las mismas circunstancias que desde finales de la primera década de este siglo ha pasado el capitalismo internacional y las consecuencias sociales que hemos conocido asociadas a esta gran crisis mundial. Así los recortes de todo tipo, salariales y de servicios públicos, el incremento del desempleo y la precarización de las condiciones de trabajo, además de tener repercusiones en el nivel de consumo privado mercantil puro, han dejado sin cobertura consumos públicos de hecho bastante restringidos desde el inicio de la era neoliberal; esto ha impulsado estrategias y estilos de vida fundamentalmente defensivos de solidaridad comunitaria, reasignación de tiempos y recursos y formas de ahorro para mantener posibilidades vitales. Lo que el Estado ya no suministra 0 en el mercado se ha puesto a un precio imposible de conseguir, se puede intentar sustituir por la vía del intercambio, la reutilización, el uso conjunto, el reparto consciente 0 la llamada al procomún y los bienes comunes, de tal forma que el consumidor del servicio puede tomar en un turno sucesivo la función de suministrador o productor, lo que hace que los roles económicos se vuelvan mucho más flexibles, horizontales y reversibles. El prosumidor colaborativo es, a la vez, un productor y consumidor activo capaz de ofrecer y recibir bienes y servicios sin que el precio sea la única razón (y el único regulador) de ese intercambio.

Directamente vinculado a lo anterior se encuentra la dimensión movimiento social que ha tomado la economía compartida y el consumo colaborativo. Revitalizando y sintetizando los repertorios y vocabularios de motivos que los movimientos sociales 
habían introducido en el ámbito del consumo un buen número de tópicos discursivos extraordinariamente difundidos —en muchos casos hasta su desgaste, degradación 0 desubstanciación-, de tal manera que a lo largo de los años del cambio de siglo vivimos un auténtico aluvión de términos entrelazados entre el activismo social y formas alternativas de consumo; como ya dijimos sostenibilidad organicidad, decrecimiento 0 solidaridad se iban asociando al consumo en el relato de grupos alternativos y minorías activas movilizadas. Pero con la crisis tanto la dimensión más retórica, como las prácticas del downsifting o limitación autoconsciente del volumen del consumo y los requerimientos mercantiles - la posibilidad de vivir mejor con menos ${ }^{4}$ — además de difundirse 0 generalizarse a públicos no históricamente movilizados se mostraron como un auténtico desafío simbólico y comunitario a la hegemonía de la financiarización absoluta del argumentario económico (liberal) dominante.

La desconfianza y hasta el rechazo ante el depredador desarrollo de las estrategias privatizadoras y mercantiles puras mantenidas durante la crisis, con sus secuelas de desposesión incremento de la desigualdad, exclusión y hasta expulsión de

4 El movimiento del downshifting, o el intento consciente de aumentar la calidad de vida simplificando el tipo de consumo y reduciendo el volumen de bienes materiales por el que sentimos atracción (a la vez que servidumbre, consiguiendo con ello aumentar nuestro tiempo liberado y aumentar la facilidad, independencia y autonomía de nuestras vidas, siempre se ha considerado como un paso previo al consumo colaborativo. Se pueden ver varias introducciones desde diferentes enfoques en español; por ejemplo: Domínguez y Robin (1997), Fresneda (1998), Wagman y Arrizabalaga (1997), Drake (2002) y Lodeiro (2008). Como siempre en estos casos, el tópico del downshifting se ha constituido desde posiciones que van desde el liberalismo desencantado de ejecutivos estresados que desean cambiar de vida hasta el libertarismo activo de seguidores de la obra de Ivan Illich 0 de movimientos ecologistas, más o menos tradicionales. Obsérvese además la coincidencia formal de enfoque y hasta de títulos entre las obras consagradas al downshifting y las más recientes dedicadas al consumo colaborativo (Cañigueral, 2014). Para el tema concurrente del decrecimiento frente a la sociedad de consumo el clásico es Serge Latouche (por ejemplo, 2012) y su versión popular relacionada con el consumo colaborativo militante Lahille (2009). los grupos sociales más vulnerables; pero también el absoluto distanciamiento de gran parte de la población con respecto a lo público (y a lo político institucional) al ser percibidos sus representantes como agentes, del poder mercantil o menos siervos de las estrategias de las grandes fuerzas financieras, la crisis de legitimidad de la público estatal más 0 menos larvada, más 0 menos abierta, a lo largo del ciclo ascendente neoliberal, se agudizaba justo cuando las soluciones políticas bloquean la capacidad redistributivas de lo público, lo corrompen o lo privatizan. Ante esta perspectiva aparece "lo común" como una vía de avance de lo social y la lógica de los bienes comunes como un discurso intelectualmente atractivo, no exento de expresiones prácticas evidentes - entre ellas del consumo colaborativo - que buscaba, muchas veces buceando en el pasado comunitario de origen precapitalista, en la tradición anarquista o en la antropología del don una vía de reconstrucción de un espacio público no estatal ante el devastador panorama de lo colectivo dejado por el imperialismo de la economía liberal convertida en santo y seña moral de lo privado y de lo público institucional ${ }^{5}$.

El consumo colaborativo irrumpió en nuestro panorama cotidiano como un proceso de racionalización social del uso de los recursos de las poblaciones y con resultados anunciados de ahorro energético, contención ecológica y enriquecimiento de la dimensión convivencial de los grupos humanos, los ejemplos sencillos, pero muy eficaces, de los trayectos compartidos, los sofás abiertos a la acogida a gente dispuesta a intercambiar, el uso

5 Para seguir la eclosión del tema asociado a ciertos aspectos del consumo colaborativo del procomún y la economía de los bienes comunes, puede verse el dossier compilado en Alonso y Piñeiro (2015), con contribuciones de especialistas fundamentales del tema en nuestro país como Rafael Ibáñez Rojo, César Rendueles, Igor Sádaba, Luis Gónzalez Reyes, Alberto Lafuente, Ángel Calle, etc.). En cuanto al tema de la definición de los bienes comunes su polémica sobre su casi tragedia 0 imposibilidad esencial, pueden verse desde diferentes perspectivas Mattei (2013) y Laín Escandell (2015). Sobre la aparición de discursos de lo común y lo colaborativo en los discursos de los movimientos sociales de la crisis pueden consultarse Alonso (2015) y Alonso, Fernández e Ibáñez Rojo (2015). 
comunitario de objetivos que habían sido pensados para el consumo individual y exclusivo — como el taladro o la podadora-, o el intercambio de objetos de segunda mano para los que no se le encontraba utilidad personal, pero sí que podían servirle a otras personas, etc., eran magníficos vehículos de comunicación y exposición pedagógica que abría un panorama alternativo a los formas de consumo altamente empresarializadas. La meteórica aparición del fenómeno en el universo internet hacía el resto, lo que había podido ser local y de proximidad, se convertía en global y a larga distancia con repercusiones prácticamente universales. Las posibilidades de articulación y coordinación de todas estas redes en plataformas temáticas coronaban la creación de un nuevo fenómeno con una potencia (por lo menos en su recepción práctica) muy superior a otras experiencias de consumo alternativo anteriores.

\section{LOS LÍMITES SOBREVENIDOS}

Sin embargo, también con una rapidez inusitada, y seguramente en parte por su éxito, los problemas de definición y propiedad de muchas de sus experiencias más emblemáticas empezaron a producirse, de tal forma que en casos como BlaBlaCar, AirBnB o Uber, por solo citar algunos bien conocidos, la polémica afloró con fuerza, no sólo parecía que los fenómenos más exitosos se empresarializaban y se acercaban a una forma de nueva mediación comercial más o menos original, en la era de las redes sociales sino que también, perdían de forma escandalosamente veloz sus connotaciones de alternativo, comunitario, de carácter principal no lucrativo, de desarrollo estrictamente horizontal y con efectos medioambientales positivos inmediatamente evaluables. Los problemas se multiplican si se abordan los asuntos de fiscalidad y regulación; cuando aparecen beneficios empresariales indiscutibles aunque sea bajo estas formas de acción coordinadas y compartidas, la obligación fiscal no puede ser eludida 0 estaremos en un caso vergonzoso y escandaloso de fraude fiscal convencional.

La seguridad, derechos y garantías jurídicas de todos los implicados en las transacciones es otro agujero negro que pronto ha tenido efectos casi explosivos, no sólo por las protestas, huelgas y prohibiciones judiciales derivadas de las acciones de colectivos que se han sentido agredidos por una competencia que consideran ilícita, desleal y clandestina (recuérdense los casos muy publicitados en el sector del taxi, el transporte urbano o por carretera, los hoteles, o los talleres de reparación de todo tipo). También han levantado sospechas y herido muchas sensibilidades, la no certeza de los derechos de restitución en caso de incumplimiento del intercambio, la poca claridad en lo que se refiere a las seguridades jurídicas asociadas a las actividades (incluida la seguridad vital de los implicados) y la muy presente posibilidad de inducir precariedad, fraude 0 economía sumergida en este proceso, dado el escaso 0 inexistente control institucional de los procesos autodeclarados como colaborativos, pero que pueden encubrir prácticas muy diversas y por ello también peligrosas. En suma, nos topamos con todas las dificultades sobrevenidas de la inexistencia de un estatuto jurídico pleno y capaz de dar respuesta institucional a un fenómeno que en estos momentos todavía se desenvuelve en el sector informal ${ }^{6}$.

6 El tema de la supervisión legal y la regulación de los consumos colaborativos es uno de los tópicos más difundidos en el debate público y con más repercusión. Este tema ha sido fundamental tanto en lo que se refiere a la consulta realizada por la Comisión Nacional del Mercado y la Competencia (CNMC), como en la encuesta sobre satisfacción, calidad y problemas declarados por los usuarios la Organización de Consumidores y Usuarios (OCU) — con la UCM y otras organizaciones de defensas del consumidor europeas-, ambas se han difundido a principios de 2016. En lo tocante a la regulación, la propia CNMC ya ha realizado un conjunto de recomendaciones sobre el marco normativo de la economía colaborativa; asimismo en la Unión Europea, el Comité Económico y Social, el Comité de Regiones y el propio Parlamento Europeo han emitido dictámenes, estudios, informes y recomendaciones variadas. En las páginas web de todos estos organismos 0 asociaciones se pueden encontrar sin dificultad toda la información, como no es el objetivo de este artículo no las reseñaremos exhaustivamente para no hacerlo demasiado prolijo, pues nuestro interés aquí no deja de ser una reflexión académica introductoria. Lo que está claro es que una vez pasado el primer entusiasmo conseguido, por ejemplo, 0 a base de difundir la declaración por la revista Time de marzo de 2011 — y otras similares - de que el consumo colaborativo sería una de las diez ideas que cambiarían el 
El optimismo sobre la economía de las plataformas y la galaxia internet también ha sido últimamente limitado y relativizado. Si nadie puede negar las espectaculares transformaciones sobre la vida y la calidad de vida de grandes grupos de la población y de que este hecho tecnológico es fundamental para la comprensión del cambio social en general y comercial en particular, es difícil derivar de ello la entrada en un universo postcapitalista (Mason, 2016), la consagración de una especie de nueva economía de la abundancia y del coste marginal cero o de la soberanía de un nuevo consumidor organizado en comunidades virtuales desafiando el poder de los antiguos oligopolios mercantiles. Como se ha argumentado con propiedad ${ }^{7}$, el solucionismo tecnológico que espera encontrar sólo en el uso de la técnica su evolución y sus aplicaciones la resolución automática de los problemas institucionales, banaliza el carácter conflictivo, complejo y adaptativo de la realidad social, de tal forma que es incapaz de reconocer que lejos de superar al capitalismo, éste llamado consumo colaborativo ha tomado una dimensión mercantil diferente —en muchos casos más potente y manipuladora-, individualizando, personalizando y ocultando las formas disciplinarias de ajuste de los grupos sociales a la producción privada y mercantil, en el nuevo control del mundo y de la vida cotidiana de las poblaciones ${ }^{8}$. El consumo colaborativo a través de internet está lleno de contradicciones, así como

mundo en nuestro siglo, se nos abre un panorama muy borroso, reflejo de la diversidad, complejidad, posibilidades y peligros que lleva aparejado el tema. Sobre esto, con diferentes enfoques véanse: Cañigeral (2014) y su muy informada página web (www.consumocolaborativo. com), así como el número monográfico preparado por el Centre de Recerca i Informació en Consum CRIC (2014), así como su página web (www.opcions.cat).

7 Es el centro de la obra de Eugeny Morozov, uno de los críticos más duros del solucionismo tecnológico y de la mitificación de las posibilidades sociales de las redes informáticas, véase Morozov (2012 y 2015.Desde el punto del análisis crítico de las organizaciones se puede ver el libro de Mendelson (2012).

8 La idea del prosumidor como nuevo agente de una forma evolucionada de capitalismo postfordista está siendo desarrollada en nuestro país por Gil García (2016), siguiendo los interesantes trabajos de orientación foucaultina de Laval y Dardot (2013). de servidumbres a los poderes tecnológicos y a los nuevos agentes hegemónicos en el universo virtual (compañías electrónicas, operadoras digitales, plataformas y buscadores virtuales, etc.), constituidos como intermediarios omnipotentes y omnipresentes que actúan además según una lógica de fondo estrictamente mercantil. Pensar que la red, también en este ámbito del consumo colaborativo, es sólo un conjunto de buenas intenciones y comportamientos altruistas es dejar pasar sin estudiar gran parte de los elementos estructurales que dominan literalmente todo este espacio donde se incrusta lo colaborativo de base informática. Carlo Ratti y Richard Sennett (2016) después de comparar la economía colaborativa actual con los elementos tradicionales de la definición consumo ostentoso del clásico de los clásicos de la sociología del consumo, Thorstein Veblen, acaban diagnosticando el presente drama de compartir: "No estamos ante un nirvana tecnológico. La economía colaborativa perturba profundamente los métodos de producción actuales, en las ciudades puede llegar a destruir el pequeño comercio y en lugar de enriquecer nuestra cultura, puede empobrecerla. Lo cual significa que debemos aprender a compartir bien".

\section{CONCLUSIÓN}

En definitiva, el consumo colaborativo abre posibilidades evidentes de cambio social y mejora de las condiciones de vida de grupos sociales que se pueden considerar mucho más que minorías activistas. En este momento nos estamos desenvolviendo todavía ante un fenómeno que se encuentra entre el movimiento social, la escasa definición institucional y su asedio por la economía mercantil. Sabemos ya de su vulnerabilidad con respecto a la expansión de nuevas formas de mercado, y por ello la necesidad de encontrar también planteamientos que relacionen estas nuevas formas de acción colectiva con los bienes y consumos públicos y no sólo con el comercio venal eminentemente privado. De todo ello se deriva la necesidad subsiguiente de regulaciones flexibles, pero eficaces y leales con todos los ciudadanos - y no sólo con los beneficiados en los intercambios directos-, así como la 
aclaración y delimitación de lo lucrativo dentro de este fenómeno que no tiene necesariamente que excluirse de antemano, pero sí controlarse para evitar efectos perversos.

Presentar el consumo colaborativo, o economía colaborativa en general como una alternativa ecológica, global y universal a las formas de asignación tradicionales, sean mercantiles o estatales es, a la vez que ingenuo, distorsionador, porque desconecta este ámbito de actividad de los mecanismos principales de producción y distribución de la riqueza, convirtiendo experiencias limitadas, pero interesantes 0 incluso formas defensivas de supervivencia y resistencia, en alternativas civilizatorias, difíciles de comprobar en la realidad y, sobre todo, de defender intelectualmente, si se presentan de manera tan ideal. Pero lo cierto es que nos encontramos ante un conjunto de prácticas a analizar y considerar tanto por sus efectos en lo social real, como por su posibilidad de abrir caminos en la construcción de una idea de lo común y de los bienes comunes que sea capaz de crear nuevas vías para el desarrollo del bienestar de las personas, en búsqueda permanente de formas activas mejores de organizar nuestra vida y nuestra convivencia.

Las contribuciones que completan esta sección abordan problemas esenciales del consumo colaborativo desde dos ángulos bien diferenciados, la de Piñeiro, Suriñach y Casadevante es una completa y clarificadora reflexión hecha desde el ámbito de los movimientos sociales y las prácticas más avanzadas socialmente del consumo colaborativo, no exenta, por cierto de una potente reflexión analítica sobre el tema. La aportación de Lain es una profunda indagación conceptual sobre la economía colaborativa y la manera de encontrar una definición no banal o puramente nominal sobre el tema de los consumos colaborativos a la luz de la sociología económica y la economía institucional. El panorama no puede ser sociológicamente más interesante.

\section{REFERENCIAS BIBLIOGRÁFICAS}

Algar, R. (2007). "Collaborative consumption", Leisure Report, abril, pp. 16-17.
Alonso, L. E., (2015). "Indignación, protesta y cooperación", en Torres Albero, C. (ed.), España 2015. Situación Social, Madrid, Centro de Investigaciones Sociológicas, pp. 1107-1113.

Alonso, L. E., Fernández Rodríguez, C. J. Ibáñez Rojo, R. (2015). "From consumerism to guilt: economic crisis and discourses about consumption in Spain": Journal of Consumer Culture, 15 (1), marzo, pp. 66-85.

Alonso, L. E. y Piñeiro, C. (eds.), (2015). El procomún y los bienes comunes, Dossieres Economistas sin Fronteras, n. ${ }^{0}$ 16, invierno, www. econsfron.org.

Belk, R. (2010). "Sharing", en Journal of Consumer Research, 36 (5), pp. 715-734.

Botsman, R. y Rogers, R. (2010). What's Mine is Yours: The Rise of Collaborative Consumption, Nueva York, Harper Collins.

Brändle, G. (2013). "Obsolescencia planeada y consumo colaborativo: ¿Tendencias contrapuestas en la sociedad de consumo actual? Comunicación presentada al XI Congreso Español de Sociología (F.E.S.), Madrid.

Cañigueral, A. (2014). Vivir mejor con menos, Barcelona, Conecta.

CRIC (Centre de Recerca i Informació en Consum) (2014). "Consumo Colaborativo: compartamos, pero bien", en Opciones n. ${ }^{0} 48$, otoño, pp. 12-24.

Domínguez, J. y Robin, V. (1997). La bolsa o la vida, Barcelona, Plaza y Janés.

Drake, J. J. (2002), Vivir más trabajar menos. Downshifting una nueva opción de vida, Barcelona, Paidós.

Felson M. y Spaeth, J. L. (1978). "Community Structure and Collaborative Consumption. A routine Activity Approach", en The American Behavioral Scientist, vol. 21, n. ${ }^{0}$ 4, pp. 614-623.

Fresneda, C. (1998). La vida simple. De los excesos de la sociedad de consumo a la búsqueda de nuevos estilos de vida, Barcelona, Planeta.

Gil García, J. (2016). "Ni consumidores ni productores: prosumidores. Una primera tipología prosumidora". Comunicación presentada al XII Congreso Español de Sociología, FES, Gijón, 29 junio-30 de julio.

Habermas, J. (1999), Problemas de legitimación en el capitalismo tardío, Madrid, Cátedra. 
Hirschman, A. 0. (2014). Más allá de la economía. Antología de ensayos, México, Fondo de Cultura Económica.

Lahille, Ph. (2009). Vivre simplement pour vivre mieux, París, Dangles.

Laín Escandell, B. (2015). "Bienes comunes, nuevos cercamientos y economía política popular", Política y Sociedad, 52 (1), pp. 99-124.

Laval, Ch., y Dardot, P. (2013). La nueva razón del mundo. Ensayo sobre la sociedad neoliberal. Barcelona, Gedisa.

Latouche, S. (2012). Salir de la sociedad de consumo, Barcelona, Octaedro.

Lodeiro, T. (2008). Consumir menos vivir mejor, Tafalla, Navarra, Txlaparta.

Mason, P. (2016). Postcapitalismo. Hacia un nuevo futuro, Barcelona, Paidós.

Mattei, U. (2013). Bienes comunes. Un manifiesto, Madrid, Trotta.

McIntosh, M. (2013). The Necessary Transition. The Journey towards the Sustainable Enterprise Economy, Londres, Greenleaf.
Mendelson, B. J. (2012). Social Media is Bullshit, Nueva York, St. Martin's Press.

Morozov, E. (2012). El desengaño de internet. Los mitos de la libertad en la red, Barcelona, Destino.

Morozov, E. (2015). La locura del solucionismo tecnológico, Buenos Aires y Madrid, Katz/Clave Intelectual.

Ratti, C. y Sennett, R. (2016). "El drama de compartir", en El País, 28 de agosto.

Sen, A. (1989). "Racionalidad, interés e identidad", en Foxley, A. y otros (eds.). Democracia, desarrollo y el arte de traspasar fronteras. Ensayos en homenaje a Albert 0. Hirschman, México, Fondo de Cultura Económica, pp. 357-367.

Shor, J. (2014). "Consumo colaborativo: una introducción", en Valor C. (ed.), pp. 7-10.

Valor, C. (ed.), (2014). Economía en colaboración, Dossieres Economistas sin Fronteras, n. ${ }^{0} 12$, febrero, www.econsfron.org.

Wagman, D. y Arrizabalaga, A. (1997). Vivir mejor con menos, Madrid, Aguilar. 
